\title{
Multiple Attributes Group Decision Making under Intuitionistic Interval-Valued Fuzzy Preference Settings
}

\author{
Lishi Zhang \\ School of Science, Dalian Ocean University, Dalian, China \\ zls@dlou.edu.cn
}

\begin{abstract}
Keywords: Group decision-making problem; Intuitionistic interval-valued fuzzy set; Intuitionistic interval-valued fuzzy number; Intuitionistic interval-valued fuzzy weighted averaging operator; Score function
\end{abstract}

\begin{abstract}
This paper studies the group decision-making problem based on interval-valued intuitionistic fuzzy preference relations.Firstly, with the IV IFWA operators, the interval-valued intuitionistic fuzzy preference relations are aggregated.Then, a novel score function which estimates the abilities that one alternative dominates the others while the alternative is dominated by all the others, the performance of the alternatives are evaluated by the score function. Finally, an illustrative numerical example is provided to demonstrate the feasibility of the proposed approach.
\end{abstract}

\section{Introduction}

Atanassov[1] introduced the concept of intuitionistic fuzzy set(IFS) which is a generalization of the concept of Zadeh's fuzzy set. The elements in an IFS are expressed in the form of ordered pairs which are presented as a membership degree and a non-membership degree respectively. Later, Atanassov and Gargov[2] generalized the intuitionistic fuzzy set(IFS) to interval-valued intuitionistic fuzzy set(IVIFS) by which the membership degree and nonmembership degree are proposed to interval numbers. With the appearance of IFS and IVIFS, they have captured more and more attention and have been put into applications successfully in many situations [3]-[15] such as management, engineering, and economics. With the complexity of the real world, the information sometimes is convenient to express by preference relations in expressing decision makers' preferences over alternatives. In such cases, decision makers may not be able to express one's preferences for alternatives exactly for the lack of background knowledge and the fuzziness and uncertainties of the information. Thus, it is acceptable to provide the decision makers's preference in terms of interval valued intuitionistic fuzzy numbers $[16,17,18,19,20]$.

The remainder of the paper is structured as follows. Section 2 recalls some basic notions and concepts about, interval-valued intuitionistic fuzzy sets and the corresponding preference relations. In Section 3, an approach to decision making under interactive interval-valued intuitionistic fuzzy preference relations settings is proposed, a new score function of alternatives is introduced. A numerical example is presented in section 4 , section 5 completes the paper.

\section{Preliminaries}

In this section, we will briefly recall some basic concepts and notions, background knowledge of interval valued intuitionistic fuzzy sets is introduced.

Definition 2 [2] Let $X$ a set, an interval valued intuitionistic fuzzy set (IVIFS) $A$ in $X$ is defined as $A=\left\{\left\langle x, \mu_{A}(x), v_{A}(x)\right\rangle \mid x \in X\right\}$

Where $\mu_{A}(x)$ and $v_{A}(x)$ with the condition $0 \leq \sup \left(\mu_{A}(x)+v_{A}(x)\right) \leq 1$, the intervals $\mu_{A}(x)$ and $v_{A}(x)$ represent, respectively, the membership degree and nonmembership degree of the element $x$ to the set $A$. For each $x \in X$, and $\mu_{A}(x)$ and $v_{A}(x)$ are closed intervals and their lower and upper end points are, respectively, denoted by. $\mu_{A L}(x)$ and $\mu_{A U}(x), v_{A L}(x)$ and $v_{A U}(x)$.Thus, an IVIFS $A$ in $X$ is expressed by $A=\left\{\left\langle x \mid\left[\mu_{A L}(x), \mu_{A U}(x)\right],\left[v_{A L}(x), v_{A U}(x)\right]\right\rangle \mid x \in X\right\}$ 
Where $0 \leq \mu_{A U}(x)+v_{A U}(x) \leq 1$.

$\mathrm{Xu}$ [11] gave the definition of IVIFN(interval-valued intuitionistic fuzzy number):An IVIFN is denoted by $\alpha=\langle[a, b],[c, d]\rangle$, where $[a, b] \subseteq[0,1],[c, d] \subseteq[0,1]$ are the interval membership degree and nonmembership degree of the element $x$ to the set $A$ with the condition $b+d \leq 1$.

\section{For IVIFSs}

$$
\begin{aligned}
& A_{1}=\left\{\left\langle x \mid\left[a_{1}(x), b_{1}(x)\right],\left[c_{1}(x), d_{1}(x)\right]\right\rangle \mid x \in X\right\}, \\
& A_{2}=\left\{\left\langle x \mid\left[a_{2}(x), b_{2}(x)\right],\left[c_{2}(x), d_{2}(x)\right]\right\rangle \mid x \in X\right\}, \\
& A=\{\langle x \mid[a(x), b(x)],[c(x), d(x)]\rangle \mid x \in X\}
\end{aligned}
$$

The following operations are defined as follows:
(1) $A_{1} \subseteq A_{2}$ if and only if for all $\forall x \in X, a_{1}(x) \leq a_{2}(x), b_{1}(x) \leq b_{2}(x), c_{1}(x) \geq c_{2}(x), d_{1}(x) \geq d_{2}(x)$,
(2) $A_{1}=A_{2}$ if and only if for all $\forall x \in X, a_{1}(x)=a_{2}(x), b_{1}(x)=b_{2}(x), c_{1}(x)=c_{2}(x), d_{1}(x)=d_{2}(x)$,
(3) $A^{c}=\{\langle x \mid[c(x), d(x)],[a(x), b(x)]\rangle \mid x \in X\}$

For an IVIFN $\alpha=\langle[a, b],[c, d]\rangle$, the score of $\alpha$ can be evaluated by the score function [11] as shown below:

$$
s(\alpha)=\frac{1}{2}(a-c+b+d)
$$

Score $s(\alpha)$ of an IVIFN reflects the degree of support of $\alpha$. The bigger the score is, the larger the IVIFN is, the score $s(\alpha) \in[-1,1]$, furthermore, the biggest one is $\langle[1,1],[0,0]\rangle$, while the smallest one is $\langle[0,0],[1,1]\rangle$. For the consideration of operations, $\mathrm{Xu}[11]$ defined some basic operational laws of IVIFNs.

\section{Definition 3[11] Let}

$$
\begin{aligned}
& \alpha_{1}=\left\langle\left[a_{1}, b_{1}\right],\left[c_{1}, d_{1}\right]\right\rangle, \alpha_{2}=\left\langle\left[a_{2}, b_{2}\right],\left[c_{2}, d_{2}\right]\right\rangle, \alpha=\langle[a, b],[c, d]\rangle \\
& \text { (1) } \alpha_{1} \wedge \alpha_{2}=\left\langle\left[\min \left\{a_{1}, a_{2}\right\}, \min \left\{b_{1}, b_{2}\right\}\right],\left[\max \left\{c_{1}, c_{2}\right\}, \max \left\{d_{1}, d_{2}\right\}\right]\right\rangle, \\
& \text { (2) } \alpha_{1} \vee \alpha_{2}=\left\langle\left[\max \left\{a_{1}, a_{2}\right\}, \max \left\{b_{1}, b_{2}\right\}\right],\left[\min \left\{c_{1}, c_{2}\right\}, \min \left\{d_{1}, d_{2}\right\}\right]\right\rangle, \\
& \text { (3) } \alpha_{1} \oplus \alpha_{2}=\left\langle\left[a_{1}+a_{2}-a_{1} a_{2}, b_{1}+b_{2}-b_{1} b_{2}\right],\left[c_{1} c_{2}, d_{1} d_{2}\right]\right\rangle, \\
& \text { (4) } \alpha_{1} \otimes \alpha_{2}=\left\langle\left[a_{1} a_{2}, b_{1} b_{2}\right],\left[c_{1}+c_{2}-c_{1} c_{2}, d_{1}+d_{2}-d_{1} d_{2}\right]\right\rangle, \\
& \text { (5) } \alpha^{c}=\langle[c, d],[a, b]\rangle .
\end{aligned}
$$

Definition 4[12] Let $\Theta$ be the set of IVIFNs, $\alpha_{j}=\left\langle\left[a_{j}, b_{j}\right],\left[c_{j}, d_{j}\right]\right\rangle(j=1,2, \ldots, n)$ be a collection of IVIFNs, and let IV IFWA I $_{\mathrm{w}} \Theta^{n} \rightarrow \Theta$, if,

IVIFWA ${ }_{w}\left(\alpha_{1}, \alpha_{2}, \ldots, \alpha_{n}\right)=w_{1} \alpha_{1} \oplus w_{2} \alpha_{2} \oplus \ldots \oplus w_{n} \alpha_{n}$

Then IVIFWA is called an interval-valued intuitionistic fuzzy weighted averaging(IVIFWA) operator of dimension $\mathrm{n}$, Where $w=\left(w_{1}, w_{2}, \cdots, w_{n}\right)^{T}$ is the weight vector of $\alpha_{i}(i=1,2, \ldots, n), w_{i} \in[0,1]$ and $\sum_{i=1}^{n} w_{i}=1$

Theorem 1[12] Let $\Theta$ be the set of IVIFNs, $\alpha_{j}=\left\langle\left[a_{j}, b_{j}\right],\left[c_{j}, d_{j}\right]\right\rangle(j=1,2, \ldots, n)$ be a collection of IVIFNs, and let IV IFWA $: \Theta^{n} \rightarrow \Theta$, if, 


$$
\operatorname{IVIFWA_{w}}\left(\alpha_{1}, \alpha_{2}, \ldots, \alpha_{n}\right)=\left\langle\left[1-\prod_{j=1}^{n}\left(1-a_{j}\right)^{w_{j}}, 1-\prod_{j=1}^{n}\left(1-b_{j}\right)^{w_{j}}\right],\left[\prod_{j=1}^{n} c_{j}^{w_{j}}, \prod_{j=1}^{n} d_{j}^{w_{j}}\right]\right\rangle
$$

Then IVIFWA is called an interval-valued intuitionistic fuzzy weighted averaging(IVIFWA) operator

In particular, if $w=(1 / n, 1 / n, \cdots, 1 / n)^{T}$, then the IVIFWA operator is reduced to an intuitionistic fuzzy averaging (IVIFA) operator of dimension $n$, which is defined as follows:

$$
I V I F W A_{w}\left(\alpha_{1}, \alpha_{2}, \ldots, \alpha_{n}\right)=\frac{1}{n} \alpha_{1} \oplus \frac{1}{n} \alpha_{2} \oplus \ldots \oplus \frac{1}{n} \alpha_{n}
$$

\section{Group decision making based on interval-valued intuitionistic fuzzy preference relations}

Let $X=\left\{x_{1}, x_{2}, \cdots, x_{n}\right\}$ be a finite set of alternatives, $E=\left\{e_{1}, e_{2}, \cdots, e_{m}\right\}$ be the set of decision makers, whose weight vector is $w=\left(w_{1}, w_{2}, \cdots, w_{m}\right)^{T}$ and $\sum_{i=1}^{m} w_{i}=1$, the expert $e_{k}(k=1,2, \cdots, m)$ inputs intuitionistic fuzzy preference value for each pair $x_{i}$ and $x_{j}(i, j=1,2, \ldots, n)$, it is shown in the matrix $R_{k}=\left(r_{i j}^{(k)}\right)_{n \times n}$

\section{The proposed algorithms}

Step 1: Utilize the IVIFA operator in Eq.(3) to aggregate all the ith row into IVIFN.

$$
r_{i}^{(k)}=I V I F A_{w}\left(r_{i 1}^{(k)}, r_{i 2}^{(k)}, \ldots, r_{i n}^{(k)}\right)
$$

Step 2: Utilize the IVIFA operator in Eq.(3) to aggregate all the ith column into IVIFN.

$$
c_{i}^{(k)}=\operatorname{IVIFA} A_{w}\left(r_{1 i}^{(k)}, r_{2 i}^{(k)}, \ldots, r_{n i}^{(k)}\right)
$$

Step 3: Utilize the IVIFWA operator in Eq.(2) to aggregate $r_{i}^{(1)}, r_{i}^{(2)}, \ldots, r_{i}^{(k)}$ into IVIFN.

$$
r_{i}=I V I F W A_{w}\left(r_{i}^{(1)}, r_{i}^{(2)}, \ldots, r_{i}^{(k)}\right)
$$

Step 4: Utilize the IVIFWA operator in Eq.(2) to aggregate $c_{i}^{(1)}, c_{i}^{(2)}, \ldots, c_{i}^{(k)}$ into IVIFN.

$$
c_{i}=I V I F W A_{w}\left(c_{i}^{(1)}, c_{i}^{(2)}, \ldots, c_{i}^{(k)}\right)
$$

Step 5: Utilize Eq.(1) to compute the score of alternatives.

$$
s\left(x_{i}\right)=s\left(r_{i}\right)-s\left(c_{i}\right)
$$

Step 6: By ranking the scores in descending order, we get optimal alternatives

\section{Case Study}

We consider a problem concerning the ranking and selecting critical criteria (adapted from [21]) which is used to assess the financial merger strategies of companies. A questionnaire which was based on a synthesis of the literature review as well as consultations and guidance from the experts employed by commercial banks in Taiwan. The investigation divided the primary goal into six categories, namely, operating performance, staff rights, customer service, bank financial composition, government policy, and risk management. These were summarized as follows [21]:(1)Operating performance $\left(x_{1}\right)$ (2)Staff rights $\left(x_{2}\right)$ (3)Customer service $\left(x_{3}\right)$ (4)Financial composition of $\operatorname{bank}\left(x_{4}\right)$ (5)Government finance policy $\left(x_{5}\right)(6)$ Risk management $\left(x_{6}\right)$.

The intuitionistic fuzzy preference values are shown as follows;

$R_{1}=\left[\begin{array}{lllllll}([0.5,0.5],[0.5,0.5]) & ([0.4,0.5],[0.2,0.3]) & ([0.2,0.4],[0.3,0.5]) & ([0.3,0.5],[0.4,0.5]) & ([0.7,0.8],[0.1,0.2]) & ([0.2,0.3],[0.5,0.7]) \\ ([0.2,0.3],[0.4,0.5]) & ([0.5,0.5],[0.5,0.5]) & ([0.1,0.3],[0.3,0.4]) & ([0.2,0.4],[0.4,0.5]) & ([0.2,0.3],[0.2,0.4]) & ([0.0,0.2],[0.5,0.6]) \\ ([0.3,0.5],[0.2,0.4]) & ([0.3,0.4],[0.1,0.3]) & ([0.5,0.5],[0.5,0.5]) & ([0.3,0.4],[0.3,0.6]) & ([0.4,0.6],[0.1,0.3]) & ([0.1,0.4],[0.3,0.5]) \\ ([0.3,0.5],[0.4,0.5]) & ([0.4,0.5],[0.2,0.4]) & ([0.3,0.6],[0.3,0.4]) & ([0.5,0.5],[0.5,0.5]) & ([0.5,0.8],[0.1,0.2]) & ([0.2,0.3],[0.6,0.7]) \\ ([0.1,0.2],[0.7,0.8]) & ([0.2,0.4],[0.2,0.3]) & ([0.1,0.3],[0.4,0.6]) & ([0.1,0.2],[0.5,0.8]) & ([0.5,0.5],[0.5,0.5]) & ([0.3,0.5],[0.4,0.5]) \\ ([0.5,0.7],[0.2,0.3]) & ([0.5,0.6],[0.0,0.2]) & ([0.3,0.5],[0.1,0.4]) & ([0.6,0.7],[0.2,0.3]) & ([0.3,0.5],[0.4,0.5]) & ([0.5,0.5],[0.5,0.5])\end{array}\right]$


$\begin{aligned} R_{2} & =\left[\begin{array}{lllllll}([0.5,0.5],[0.5,0.5]) & ([0.3,0.5],[0.1,0.3]) & ([0.3,0.4],[0.2,0.5]) & ([0.4,0.5],[0.3,0.4]) & ([0.5,0.8],[0.0,0.2]) & ([0.3,0.5],[0.4,0.5]) \\ ([0.1,0.3],[0.3,0.5]) & ([0.5,0.5],[0.5,0.5]) & ([0.1,0.3],[0.3,0.4]) & ([0.3,0.4],[0.2,0.5]) & ([0.1,0.2],[0.3,0.5]) & ([0.2,0.5],[0.3,0.4]) \\ ([0.3,0.4],[0.2,0.5]) & ([0.2,0.3],[0.0,0.3]) & ([0.5,0.5],[0.5,0.5]) & ([0.2,0.4],[0.4,0.5]) & ([0.5,0.7],[0.1,0.2]) & ([0.3,0.4],[0.4,0.6]) \\ ([0.3,0.4],[0.4,0.5]) & ([0.2,0.5],[0.3,0.4]) & ([0.4,0.5],[0.2,0.4]) & ([0.5,0.5],[0.5,0.5]) & ([0.4,0.7],[0.2,0.3]) & ([0.0,0.2],[0.5,0.7]) \\ ([0.2,0.3],[0.6,0.7]) & ([0.3,0.5],[0.1,0.3]) & ([0.1,0.2],[0.5,0.7]) & ([0.2,0.3],[0.4,0.7]) & ([0.5,0.5],[0.5,0.5]) & ([0.2,0.3],[0.3,0.6]) \\ ([0.5,0.6],[0.2,0.4]) & ([0.3,0.4],[0.2,0.3]) & ([0.4,0.6],[0.3,0.4]) & ([0.5,0.7],[0.0,0.2]) & ([0.3,0.6],[0.2,0.3]) & ([0.5,0.5],[0.5,0.5])\end{array}\right] \\ R_{3}= & \left(\begin{array}{llllll}([0.5,0.5],[0.5,0.5]) & ([0.5,0.7],[0.1,0.2]) & ([0.3,0.5],[0.2,0.4]) & ([0.2,0.3],[0.3,0.5]) & ([0.5,0.8],[0.0,0.2]) & ([0.3,0.5],[0.4,0.5]) \\ ([0.1,0.2],[0.5,0.7]) & ([0.5,0.5],[0.5,0.5]) & ([0.3,0.4],[0.4,0.6]) & ([0.1,0.3],[0.2,0.5]) & ([0.2,0.4],[0.3,0.5]) & ([0.2,0.3],[0.4,0.7]) \\ ([0.2,0.4],[0.3,0.5]) & ([0.3,0.4],[0.4,0.6]) & ([0.5,0.5],[0.5,0.5]) & ([0.4,0.5],[0.3,0.5]) & ([0.3,0.6],[0.1,0.2]) & ([0.2,0.3],[0.3,0.4]) \\ ([0.3,0.5],[0.2,0.3]) & ([0.2,0.5],[0.1,0.3]) & ([0.3,0.5],[0.4,0.5]) & ([0.5,0.5],[0.5,0.5]) & ([0.4,0.5],[0.2,0.4]) & ([0.4,0.6],[0.2,0.3]) \\ ([0.0,0.2],[0.5,0.8]) & ([0.3,0.5],[0.2,0.4]) & ([0.1,0.3],[0.4,0.6]) & ([0.2,0.4],[0.4,0.5]) & ([0.5,0.5],[0.5,0.5]) & ([0.4,0.5],[0.3,0.5]) \\ ([0.4,0.5],[0.3,0.5]) & ([0.4,0.7],[0.2,0.3]) & ([0.3,0.4],[0.2,0.3]) & ([0.2,0.3],[0.4,0.6]) & ([0.3,0.5],[0.4,0.5]) & ([0.5,0.5],[0.5,0.5])\end{array}\right]\end{aligned}$

Furthermore, by computing, we get that

$$
S\left(x_{1}\right)=0.6453, S\left(x_{2}\right)=-0.7662, S\left(x_{3}\right)=0.4284, S\left(x_{4}\right)=-0.1257, S\left(x_{5}\right)=-0.9713, S\left(x_{6}\right)=0.8499
$$

The order of scores are listed as follows

$$
A_{6} \succ A_{1} \succ A_{3} \succ A_{4} \succ A_{2} \succ A_{5}
$$

The most desirable is $A_{6}$. This result is in agreement with the one obtained in [21].

\section{References}

[1] K.Atanassov, Intuitionistic fuzzy set, Fuzzy Sets Syst. 20(1986) 87-96.

[2] K. Atanassov, G. Gargov, Interval-valued intuitionistic fuzzy set, Fuzzy Sets Syst. 31(1989)343-349.

[3] Z.P.Chen,W.Yang,A new multiple attribute group decision making method in intuitionistic fuzzy setting, Appl. Math. Model. 35(2011)4424-4437.

[4] S. M. Chen, J. M. Tan, Handling multicriteria fuzzy decision making problems based on vague set theory, Fuzzy Sets Syst. 67 (2)(1994) 163-172.

[5] G.W.Wei, Gray relational analysis method for intuitionistic fuzzy multiple attribute decision making, Expert.Syst.Appl.38(2011)11671-11677.

[6] J.Ye, Fuzzy decision-making method based on the weighted correlation coefficient under intuitionistic fuzzy environment, Eur.J.Oper.Res. 205(2010) 202-204.

[7] J.Ye, Multicriteria fuzzy decision-making method using entropy weights-based correlation coefficients of intervalvalued intuitionistic fuzzy sets, Appl.Math.Model.34(2010) 3864-3870.

[8] Z.S.Xu, Intuitionistic fuzzy aggregation operators, IEEE Trans. Fuzzy Syst.15(2007) 1179-1187.

[9] Z.S.Xu,A deviation-based approach to intuitionistic fuzzy multiple attribute group decision making, Group Decis. Negot.19 (2010)57-76.

[10] Z.S.Xu, M.M.Xia, Induced generalized intuitionistic fuzzy operators, Knowl. Based. Syst. 24(2011)197-209.

[11] Z.S.Xu, R.R.Yager, Some geometric aggregation operators based on intuitionistic fuzzy sets, Int.J.General Syst. 35(2006)417-433.

[12] Z.S.Xu, Methods for aggregating interval-valued intuitionistic fuzzy information and their application to decision making, Control Decisi. 22 (2)(2007)215-219.

[13] S. Z. Zeng, W. H. Su, Intuitionistic fuzzy ordered weighted distance operator, Knowl. Based. Syst. 24(2011)1224-1232.

[14] S.M.Chen, C.H.Chang,A novel similarity measure between Atanassov's intuitionistic fuzzy sets based on transformation techniques with applications to pattern recognition,Inf.Sci.291 (2015)96-114.

[15] S.M.Chen, C.H.Chang, Fuzzy ultiattribute decision making based on transformation techniques of intuitionistic fuzzy values and intuitionistic fuzzy geomeric averaging operators, Inf.Sci. 352-353(2016)133-149.

[16] Z.S.Xu,A method for estimating criteria weights from intuitionistic preference relations, Fuzzy 
Inform.Eng.1(2009)79-89.

[17] Z.W.Gong, Least-square method to priority of the fuzzy preference relations with incomplete information, Int.J.Approx.Reasoning.47(2008)258-264.

[18] T.C.Wang,Y.L.Lin, Applying the consistent fuzzy preference relations to select merger strategies for commercial banks in new financial environments, ExpertSyst.Appl.36(2009) 7019-7026.

[19] S.Z.Zeng,W.H.Su, L.R.Sun,A method based on similarity measures for interactive group decision making with intuitionistic fuzzy preference relations, Appl. Math. Model.37(2013) 6909-6917.

[20] S.P.wan, F.Wang, L.Y.Dong,A group making method with interal valued fuzzy preference relations based on the geometric consistency, Inf. Fusion. 40(2018)87-100.

[21] Z.S.Xu, A method based on distance measure for interval-valued intuitionistic fuzzy group decision making, Inf. Sci. 180(2010)181-190. 